\title{
Excretion of PFOA and PFOS in Male Rats During a Subchronic Exposure
}

\author{
Lin Cui · Chun-yang Liao • Qun-fang Zhou • \\ Tong-mei Xia $\cdot$ Zhao-jun Yun • \\ Gui-bin Jiang
}

Received: 29 January 2009/Accepted: 5 May 2009/Published online: 26 May 2009

(C) Springer Science+Business Media, LLC 2009

\begin{abstract}
Perfluorinated compounds (PFCs), a class of synthetic surfactants that are widely used, have become global environmental contaminants because of their high persistence and bioaccumulation. An increasing number of studies have described the pharmacokinetics of PFCs following in vivo exposure, however, few papers have focused on the excretion of these compounds during a period of consecutive exposure. In this study, the excretions of perfluorooctanoic acid (PFOA) and perfluorooctane sulfonate (PFOS) in male Sprague-Dawley rats gavaged consecutively for 28 days were investigated and compared. The faster elimination rate in urine compared to feces indicated that urinary excretion is the primary clearance route in rats for either PFOA or PFOS. During the first $24 \mathrm{~h}$ after administration of PFOA (5 and $20 \mathrm{mg} /$ $\mathrm{kg}$ body weight/day), about $24.7-29.6 \%$ of the oral dose was excreted through urine and feces, while for PFOS, the excretion amounts were only $2.6-2.8 \%$ of the total gavaged doses ( 5 and $20 \mathrm{mg} / \mathrm{kg}$ body weight/day). The excretion rates of both PFCs increased with increasing exposure doses. The higher elimination rate of PFOA through excretion indicated its lower accumulation in rats, thus inducing possible lower toxicities compared to PFOS.
\end{abstract}

L. Cui · C. Liao · Q. Zhou · Z. Yun · G. Jiang $(\bowtie)$

State Key Laboratory of Environmental Chemistry and Ecotoxicology, Research Center for Eco-Environmental Sciences, Chinese Academy of Sciences, Beijing 100085, China e-mail: gbjiang@rcees.ac.cn

\section{T. Xia}

Department of Biology, School of Applied Science, University of Science and Technology Beijing, Beijing 100083, China
Due to their unique physicochemical properties, perfluorinated compounds (PFCs), such as perfluorooctanoic acid (PFOA) and perfluorooctane sulfonate (PFOS), have been widely used in a diverse variety of industrial and commercial products (Giesy and Kannan 2002). Previous investigations have reported the widespread presence of perfluorinated acid and related fluorochemicals in wild animals and blood of the general population (Giesy and Kannan 2001; Kannan et al. 2004). Their persistence, high bioaccumulation, and potential toxicity in organisms have attracted much attention to this class of compounds, resulting in the withdrawal of PFOS from the marketplace (Lau et al. 2007).

Increasing numbers of studies have indicated the toxic effects caused by this kind of peroxisome proliferators in exposed laboratory animals (Seacat et al. 2002; 2003). PFOA and PFOS caused reductions in body weight, decreases in weight gain rate, and interference in lipid metabolism in rats (Lau et al. 2007; Luebker et al. 2002). The liver is considered the primary target organ for both acute and chronic exposure of the above two fluorochemicals, and their hepatotoxicity in rodents has been extensively studied, which mainly includes increased liver weight, induction of hepatocellular hypertrophy and lipid vacuolation, increased incidence of hepatocellular adenoma, and hypocholesterolemia (Biegel et al. 2001; Butenhoff et al. 2002; Seacat et al. 2003). Additionally, the potential neurotoxicity, genotoxicity, molecular toxicity, and reproductive and developmental toxicities of PFOA and PFOS were demonstrated in recent toxicological studies (Austin et al. 2003; Guruge et al. 2006; Kennedy et al. 2004; Lau et al. 2004; Ren et al. 2008). The pharmacokinetics of PFCs in animals has received increasing attention, which is of fundamental significance with regard to their potential biological effects. Previous 
pharmacokinetic studies on PFOA and PFOS were mostly conducted on mammals (rats, dogs, and monkeys), birds (Newsted et al. 2006; Yoo et al. 2009), and humans (Lau et al. 2004; Kudo and Kawashima 2003). The research found that both of them were well absorbed after ingestion. More than $95 \%$ of the total carbon-14 was absorbed within $24 \mathrm{~h}$ after a single oral dose by gavage of ${ }^{14} \mathrm{C}$-PFOA or ${ }^{14}$ C-PFOS (Gibson and Johnson 1979; Johnson et al. 1979). Moreover, a relatively pronounced gender difference in PFOA elimination has been observed in several animals, such as rats and beagle dogs, but not in mouse, primate, or human (Kemper 2003; Kudo and Kawashima 2003). For example, the serum elimination half-lives of PFOA were approximately 5.68 and 0.08 days in male and female rats, respectively, following intravenous (iv) administration (Kudo et al. 2002). By exposing beagle dogs, Hanhijärvi (1988) calculated plasma half-lives of 473 and $541 \mathrm{~h}$ in two males and 202 and $305 \mathrm{~h}$ in two females following a single iv injection. A universally accepted viewpoint is that estradiol promotes urinary excretion of PFOA, whereas testosterone decreases its excretion (Vanden Heuvel et al. 1992; Ylinen et al. 1989). Compared to PFOS, striking differences among species existed in the pharmacokinetics of PFOA. The combined properties of rapid absorption and poor elimination could contribute to the bioaccumulation of PFOA or PFOS. It has been determined that the accumulation of PFOA in tissues of rodents, as well as PFOS, is principally in liver, kidney, and blood (Johnson et al. 1979; Ylinen et al. 1990).

To date, limited toxicological studies are available on the examination of excretion of PFCs under consecutive exposure. In light of the ubiquity and persistence of PFCs in both the environment and organisms, the current study was undertaken to compare the excretion of PFOA versus PFOS in male Sprague-Dawley (SD) rats during a 28-day subchronic exposure. The contents of these two perfluorinated compounds in urine and feces were determined, and the influence of different oral doses ( 5 and $20 \mathrm{mg} / \mathrm{kg} /$ day) on the clearance of PFOA and PFOS is also discussed.

\section{Materials and Methods}

\section{Reagents}

Pentadecafluorooctanoic acid (PFOA; 96\%) and perfluorooctanesulfonate potassium (PFOS; 98\%) were from Sigma-Aldrich (St. Louis, MO, USA). Methyl tert-butyl ether (MTBE; 99\%) was obtained from Acros (Morris Plains, NJ, USA). Tetrabutylammonium (TBA; 97\%) hydrogen sulfate solution, $0.5 \mathrm{M}$, purchased from Alfa Aesar and adjusted to $\mathrm{pH} 10.0$ with sodium hydroxide and $0.25 \mathrm{M}$ sodium carbonate buffer solution $\left(\mathrm{Na}_{2} \mathrm{CO}_{3} /\right.$
$\mathrm{NaHCO}_{3}$; analytical reagent grade), were prepared with ultrapure water from Milli-Q Advantage A10 system (Millipore, Milford, MA, USA). The internal standard, sodium perfluoro-1-[1,2,3,4- $\left.{ }^{13} \mathrm{C}_{4}\right]$ octanesulfonate (MPFOS; ${ }^{13} \mathrm{C}_{4}^{12} \mathrm{C}_{4} \mathrm{~F}_{17} \mathrm{SO}_{3} \mathrm{Na}$; chemical purity, $\geq 98 \%$; isotopic purity, $\geq 99 \%$ ), was purchased from Wellington Laboratories (Ontario, Canada) and the work solution at the concentration of $100 \mathrm{ng} / \mathrm{mL}$ was prepared in HPLC-grade methanol (J. T. Baker, Phillipsburg, NJ, USA). The solutions described above were stored under dark conditions at $4^{\circ} \mathrm{C}$ and adjusted to room temperature just before use. Dosing preparations and concentration verification followed the methods published in our previous paper (Cui et al. 2009). Ultrapure water was used to dissolve the dosed chemicals. All the oral administration solutions of PFOA and PFOS were prepared daily in ultrapure water before dosing by dissolving adequate amounts of the chemicals. Throughout the 28-day period, all oral administration solutions of PFOA and PFOS were found to be at the nominal concentrations (with variation $<5 \%$ ), homogeneous, and stable, and neither PFOA nor PFOS was detected in ultrapure water.

\section{Animals and Husbandry}

All experiments in this research complied with the regulations and principles of the Animal Care \& Welfare Committee, Institute of Materia Medica, Chinese Academy of Medical Sciences \& Peking Union Medical College (CAMS\&PUMC).

Male SD rats (specific pathogen-free grade; 190-210 g) were obtained from Beijing Vital River Experiment Animal Technology Ltd. (Beijing) and were approximately 2 months of age at the beginning of the administration. The animal room was maintained at a temperature of $22-24^{\circ} \mathrm{C}$ and a relative humidity of $40-60 \%$ and was artificially illuminated (fluorescent light) with a 12-h light/dark cycle. Certified laboratory chow and sterilized water were available ad libitum throughout the experiment. All animals were singly housed in stainless-steel wire-mesh cages suspended above cage boards and were acclimatized to the experimental environment for at least 1 week prior to exposure. The health condition of the animals was evaluated by adequate body weight gain and freedom from clinical signs of disease, injury, or other potential confounding variables.

\section{Experimental Design}

After the quarantine period, 50 rats were randomly divided into five groups (10 per group), which were individually administered two PFC solutions by gavage exposure protocol (i.e., intragastric intubation) at doses of 0 (called G0), 
$5 \mathrm{mg}$ PFOA/kg/day (G1), $20 \mathrm{mg}$ PFOA/kg/day (G2), $5 \mathrm{mg}$ PFOS/kg/day (G3), and $20 \mathrm{mg}$ PFOS/kg/day (G4), at a standard volume of $10 \mathrm{~mL} / \mathrm{kg}$ body weight, once a day, 7 days per week, for 4 weeks. G0, receiving ultrapure water only, was set as the control group. Individual daily gavage volumes were calculated based on the latest body weights and the drugs were administered at essentially the same time everyday, so the expected dosages of the chemicals were obtained by the rats everyday.

Urine and fecal samples were collected after daily gavage with PFCs by moving rats to standard metabolism cages overnight for 24-h intervals on the following day: prior to dose administration (Day 0; baseline), on Day 1 (0-24 h postdose), and on Days 3, 5, 7, 10, 14, 18, 21, 24, and 28. For the collection of urine, the conduction pipes of the metabolism cages were directly plugged into the glass bottles with cover gaskets. Ice bags surrounding the glass bottles were replaced every $8 \mathrm{~h}$ (three times per day) to keep the collected urine at low temperatures. The volume of each urine sample and the weight of each fecal sample were measured, and the samples were marked with an identification number and stored at $-40^{\circ} \mathrm{C}$ until chemical analysis.

\section{Analytical Procedure for PFCs in Urinary and Fecal Samples}

The analytical procedure for PFOA and PFOS in urinary and fecal samples was similar to that described by Hansen et al. (2001), with some modifications in the sample pretreatment. In brief, a small portion of the urinary sample or fecal homogenate (prepared with 9 vol of water) was diluted with ultrapure water in a suitable proportion. After spiking with $50 \mu \mathrm{L}(100 \mathrm{ng} / \mathrm{mL})$ MPFOS, $1 \mathrm{~mL}$ of sample dilution was mixed thoroughly with $2 \mathrm{~mL}$ of $0.25 \mathrm{M}$ sodium carbonate buffer solution and $1 \mathrm{~mL}$ of $0.5 \mathrm{M} \mathrm{TBA}(\mathrm{pH} 10.0)$, in a $15-\mathrm{mL}$ polypropylene (PP) tube. After the addition of MTBE $(5 \mathrm{~mL})$, the mixture was shaken for extraction and centrifuged for separation, and then the organic aliquot (MTBE) was transferred to a new PP tube. The extraction and separation were repeated twice as described above. The combined organic extract was evaporated under nitrogen to dryness, resuspended in $0.5 \mathrm{~mL}$ of methanol, and extracted on a preconditioned solid-phase extraction (SPE) cartridge (Oasis HLB; $200 \mathrm{mg}, 6 \mathrm{cc}$; Waters, USA). Elution was performed with $10 \mathrm{~mL}$ of methanol and the final extract was concentrated to $1 \mathrm{~mL}$.

Analysis of target analytes was accomplished using a high-performance liquid chromatography-electrospray tandem mass spectrometry system (HPLC/ESI-MS/MS; API 3200 triple quadruple mass spectrometer configured with ESI; ABI, USA), according to the method described previously (Cui et al. 2009). Separation of PFOA and PFOS was achieved on an analytical column (Capcell Pak UG120 $\mathrm{C}_{18}$ Column; 3- $\mu \mathrm{m}$ particle diameter, $50 \mathrm{~mm} \times 2.0$ $\mathrm{mm}$ i.d.; SHIDSEIDO, Japan) with an injection aliquot of $10 \mu \mathrm{L}$. MPFOS was used for quantification, and the monitored ion pair transitions (parent ion $\rightarrow$ daughter ion) for confirmation of PFOA, PFOS, and MPFOS were $412.8 \rightarrow$ $369.0,498.8 \rightarrow 79.9$, and $502.9 \rightarrow 80.0$, respectively.

Concentrations of the target analytes were quantified using a standard calibration curve in the range of $0.05-50 \mathrm{ng} /$ $\mathrm{mL}$, and the internal standard (5 $\mathrm{ng} / \mathrm{mL}$ MPFOS) was used. The limits of quantification (LOQs) were $150 \mathrm{pg} / \mathrm{mL}$ for PFOA and $110 \mathrm{pg} / \mathrm{mL}$ for PFOS, respectively. Procedural blanks were carried out for each batch of samples and no analyte of interest was detected in any blank groups, proving no potential sources of instrumental and procedural contamination. The matrix recoveries of PFOA and PFOS certified the feasibility of the method; they were in the range of 101.4-117.3\% and 96.8-110.1\% ( $n=4$ each), respectively.

\section{Data Analyses and Statistics}

The mass contents of the two target PFCs, PFOA and PFOS, in urine and feces sampled at the appointed timepoints, calculated according to Eqs. 1 and 2, were more effective for comparing the factual excretion conditions in rats than using the concentrations. All data are expressed as mean \pm SE. One-way ANOVA was used to compare the differences between groups and different exposure durations. $p<0.05$ was regarded as a statistically significant difference.

PFC content in urine $(\mathrm{mg})=[\mathrm{PFC}$ concentration in urine $(\mu \mathrm{g} / \mathrm{mL}) \times$ Urine volume $(\mathrm{mL})] / 1000$

PFC content in feces $(\mathrm{mg})=[\mathrm{PFC}$ concentration in feces $(\mu \mathrm{g} / \mathrm{g}) \times$ Feces weight $(\mathrm{g})] / 1000$ 


\section{Results and Discussion}

Urinary and Fecal Excretion in Rats

The toxicological effects of PFOA and PFOS based on body weight evaluation, histological observation, and chemical analysis were described previously (Cui et al. 2009). Urinary and fecal excretions of these two typical perfluorocompounds were studied during a subchronic exposure in the present work. Similar to the normal rats in the control group (G0), the volume of urine and weight of feces excreted by the exposed rats (Table 1) in G1, G2, and G3 increased with body weight gains. No significant difference in excretion was observed among the above groups. However, the urinary and fecal excretion of the rats in G4 obviously decreased due to anorexia, and it was difficult to collect in the latter stage of exposure. The amounts of food and water ingested in each group were correlated with those of fecal and urinary excretion.
Elimination of PFOA in Rats

Urine

The average amounts of PFOA in the urine, for both the low- and the high-dose groups, during the entire exposure period are shown in Fig. 1a, b. In general, there was an upward trend for the urinary excretion contents of PFOA under conditions of administration of 5 and $20 \mathrm{mg} / \mathrm{kg} /$ day (Fig. 1a, b). G1 and G2 both showed their maximum at the end of treatment (Day 28): 1.200 and $9.077 \mathrm{mg}$, respectively. A similar sharp increase in urinary PFOA levels occurred in both G1 and G2 in the early stage of exposure (Week 1), indicating that efficient elimination of xenobiotics took place in exposed rats at the beginning of metabolism. The increase in G1 slowed down in the subsequent days and lasted until the end of the test, indicating that the urinary excretion of PFOA reached a relatively steady state. However, another faster rate of increase was

Table 1 Urine volume and feces weight excreted by the rats in each group during the 28-day exposure period: mean $\pm \mathrm{SE}$

\begin{tabular}{|c|c|c|c|c|c|}
\hline & \multicolumn{5}{|c|}{ Daily exposure dose by gavage } \\
\hline & $0(\mathrm{G} 0)$ & $\begin{array}{l}5 \mathrm{mg} \text { PFOA/ } \\
\mathrm{kg} \mathrm{bw} \mathrm{(G1)}\end{array}$ & $\begin{array}{l}20 \mathrm{mg} \text { PFOA/ } \\
\mathrm{kg} \text { bw (G2) }\end{array}$ & $\begin{array}{l}5 \mathrm{mg} \text { PFOS/ } \\
\mathrm{kg} \mathrm{bw} \mathrm{(G3)}\end{array}$ & $\begin{array}{l}20 \mathrm{mg} \text { PFOS/ } \\
\mathrm{kg} \text { bw (G4) }\end{array}$ \\
\hline \multicolumn{6}{|c|}{ Urine volume $(\mathrm{mL})$} \\
\hline Day 1 & $14.1 \pm 2.3$ & $14.0 \pm 2.2$ & $13.3 \pm 2.3$ & $13.8 \pm 2.6$ & $14.0 \pm 2.8$ \\
\hline Day 3 & $14.3 \pm 3.6$ & $14.2 \pm 3.8$ & $13.6 \pm 4.2$ & $13.9 \pm 2.5$ & $14.0 \pm 2.1$ \\
\hline Day 5 & $14.5 \pm 3.1$ & $14.4 \pm 2.8$ & $13.7 \pm 3.7$ & $14.0 \pm 2.8$ & $14.1 \pm 3.2$ \\
\hline Day 7 & $14.8 \pm 2.9$ & $14.6 \pm 1.9$ & $13.8 \pm 3.4$ & $14.2 \pm 4.7$ & $14.1 \pm 1.4$ \\
\hline Day 10 & $15.4 \pm 3.8$ & $15.0 \pm 4.6$ & $14.1 \pm 5.3$ & $14.8 \pm 1.9$ & $14.0 \pm 3.3$ \\
\hline Day 14 & $16.0 \pm 3.4$ & $15.9 \pm 2.7$ & $14.8 \pm 4.8$ & $15.5 \pm 2.9$ & $13.8 \pm 2.9$ \\
\hline Day 18 & $17.1 \pm 4.2$ & $16.5 \pm 2.5$ & $15.5 \pm 3.8$ & $16.2 \pm 3.8$ & $13.4 \pm 5.1$ \\
\hline Day 21 & $18.1 \pm 3.7$ & $17.2 \pm 2.1$ & $16.1 \pm 3.2$ & $16.9 \pm 5.5$ & $\mathrm{NS}^{\mathrm{a}}$ \\
\hline Day 24 & $18.9 \pm 5.6$ & $17.6 \pm 4.1$ & $16.6 \pm 4.4$ & $17.3 \pm 4.2$ & NS \\
\hline Day 28 & $19.6 \pm 5.4$ & $18.1 \pm 3.3$ & $17.1 \pm 4.6$ & $17.6 \pm 4.5$ & NS \\
\hline \multicolumn{6}{|c|}{ Feces weight (g) } \\
\hline Day 1 & $8.18 \pm 1.31$ & $7.95 \pm 0.99$ & $7.15 \pm 1.39$ & $7.55 \pm 0.86$ & $7.91 \pm 1.12$ \\
\hline Day 3 & $8.64 \pm 1.47$ & $8.08 \pm 0.97$ & $7.54 \pm 0.96$ & $7.78 \pm 1.24$ & $8.01 \pm 0.83$ \\
\hline Day 5 & $9.09 \pm 1.77$ & $8.23 \pm 1.26$ & $7.75 \pm 1.01$ & $8.00 \pm 1.16$ & $8.09 \pm 1.19$ \\
\hline Day 7 & $9.54 \pm 1.58$ & $8.36 \pm 1.64$ & $7.78 \pm 0.98$ & $8.23 \pm 1.73$ & $8.18 \pm 1.74$ \\
\hline Day 10 & $10.8 \pm 1.9$ & $9.98 \pm 1.71$ & $7.94 \pm 1.27$ & $9.62 \pm 1.98$ & $7.92 \pm 2.47$ \\
\hline Day 14 & $12.1 \pm 2.9$ & $11.6 \pm 2.5$ & $9.62 \pm 1.52$ & $11.0 \pm 2.9$ & $7.65 \pm 3.91$ \\
\hline Day 18 & $14.2 \pm 3.3$ & $13.1 \pm 4.0$ & $11.1 \pm 2.2$ & $12.4 \pm 2.4$ & $6.77 \pm 3.52$ \\
\hline Day 21 & $16.4 \pm 4.5$ & $14.5 \pm 3.9$ & $12.3 \pm 4.0$ & $13.9 \pm 3.5$ & $5.89 \pm 4.66^{\mathrm{b}}$ \\
\hline Day 24 & $18.0 \pm 3.2$ & $15.4 \pm 5.1$ & $13.5 \pm 5.3$ & $14.7 \pm 4.2$ & $3.45 \pm 2.78^{\mathrm{c}}$ \\
\hline Day 28 & $19.6 \pm 4.8$ & $16.4 \pm 4.8$ & $14.3 \pm 4.6$ & $15.4 \pm 5.5$ & NS \\
\hline
\end{tabular}

Note: $b w$ body weight, NS $n \geq 5$

${ }^{a} N S$ no urine sample was available in the $20 \mathrm{mg}$ PFOS/kg bw (G4) dose group after Day 18 because the dying rats could barely excrete in articulo mortis, while few fecal samples could be obtained from the surviving rats until Day 24

${ }^{\mathrm{b}} n=4$; only four rats alive

c $n=2$; only two rats alive 

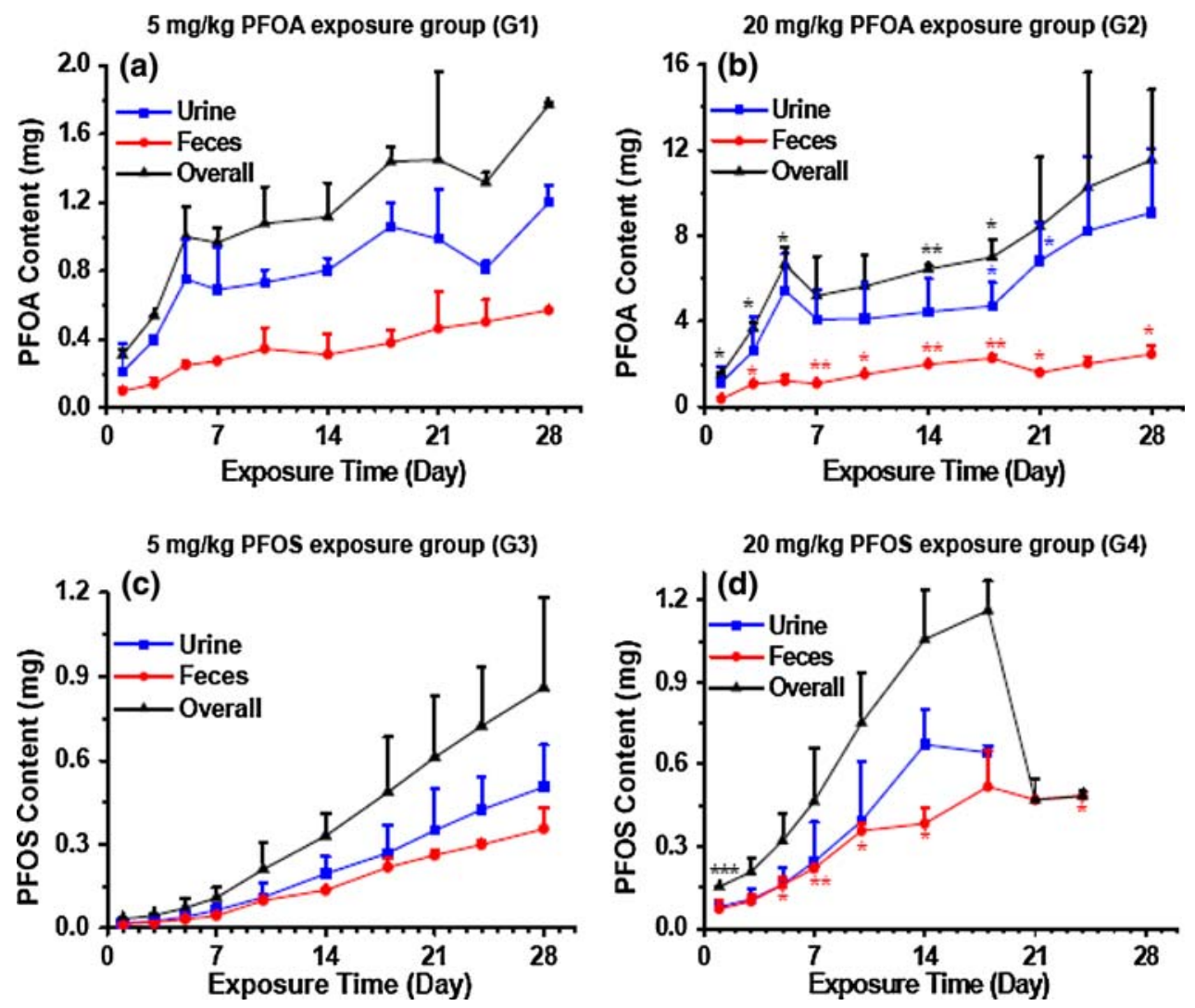

Fig. 1 PFOA or PFOS contents $(\mathrm{mg})$ in the urinary, fecal, and overall excretion of male rats from different treatment groups during the 28day exposure period: (a) $5 \mathrm{mg} / \mathrm{kg}$ body weight (bw)/day PFOA (G1); (b) $20 \mathrm{mg} / \mathrm{kg}$ bw/day PFOA (G2); (c) $5 \mathrm{mg} / \mathrm{kg}$ bw/day PFOS (G3); (d) $20 \mathrm{mg} / \mathrm{kg}$ bw/day PFOS (G4). Data are expressed as mean $\pm \mathrm{SE}$ $(n \geq 5)$. * $p<0.05$, ** $p<0.01$, and $* * * p<0.001$; comparison between the low dose ( $5 \mathrm{mg} / \mathrm{kg}$ bw/day) and the high dose $(20 \mathrm{mg} / \mathrm{kg}$

observed in G2 from Day 18 (Fig. 1b). The PFOA contents in urinary excretion in $\mathrm{G} 2$ increased greatly in the latter period of the experiment.

Urinary excretion represents a portion of the systemically absorbed oral dose of substances eliminated through urine. Excretion rates of PFCs (i.e., the ratios of the target chemical amounts in the excretion to the actual gavage doses, expressed as percentages) are regarded as important indexes to evaluate their elimination capability (Kudo et al. 2001). In the first $24 \mathrm{~h}$ after administration, the average daily urinary excretion rates of PFOA were $17.5 \%$ for G1 and $22.0 \%$ for $\mathrm{G} 2$, in agreement with the reported value of $20 \%$ in male rats after an iv injection (Johnson and Ober 1980). However, upward trends in urinary excretion rates of G1 and G2 (Fig. 2a) continued throughout the exposure period, similar to the change in PFOA contents in the urinary excretion. All the ratios at the chosen timepoints were much higher in G2 than in G1, suggesting that PFOA elimination through the urine was relatively faster in G2

bw/day) at the same timepoint (one-way ANOVA). Concentrations of PFOA and PFOS in the urine and fecal samples of the control group (G0) were not detected (lower than the LOQs). No urine sample was available in the $20 \mathrm{mg} / \mathrm{kg}$ bw/day PFOS dose group (G4) after Day 18 because the dying rats could barely excrete in articulo mortis, while few fecal samples could be obtained from the surviving rats until Day $24(n=4$ on Day 21 and $n=2$ on Day 24)

than in G1. Further, this indicated that urinary excretion of PFOA was accelerated by increased gavage dose.

\section{Feces}

Figure 1a, b also present the mean fecal excretion contents of PFOA during treatment. As with urinary excretion, fecal excretion in G1 and G2 (Fig. 1a) also maintained an upward trend throughout the study; the maximum values of 0.571 and $2.470 \mathrm{mg}$ were observed on the final day of exposure (Day 28) in G1 and G2, respectively. Similar to the relationship of urinary excretion between $\mathrm{G} 1$ and G2, the fecal excretion amounts of PFOA in G2 were also significantly higher than those in G1. According to the physiologically motivated pharmacokinetic model for rats exposed to toxic substances, fecal excretion consists of systemically absorbed toxicants present in the digestive tract and the fraction of unabsorbed toxicants (Tan et al. 2008); thus, 


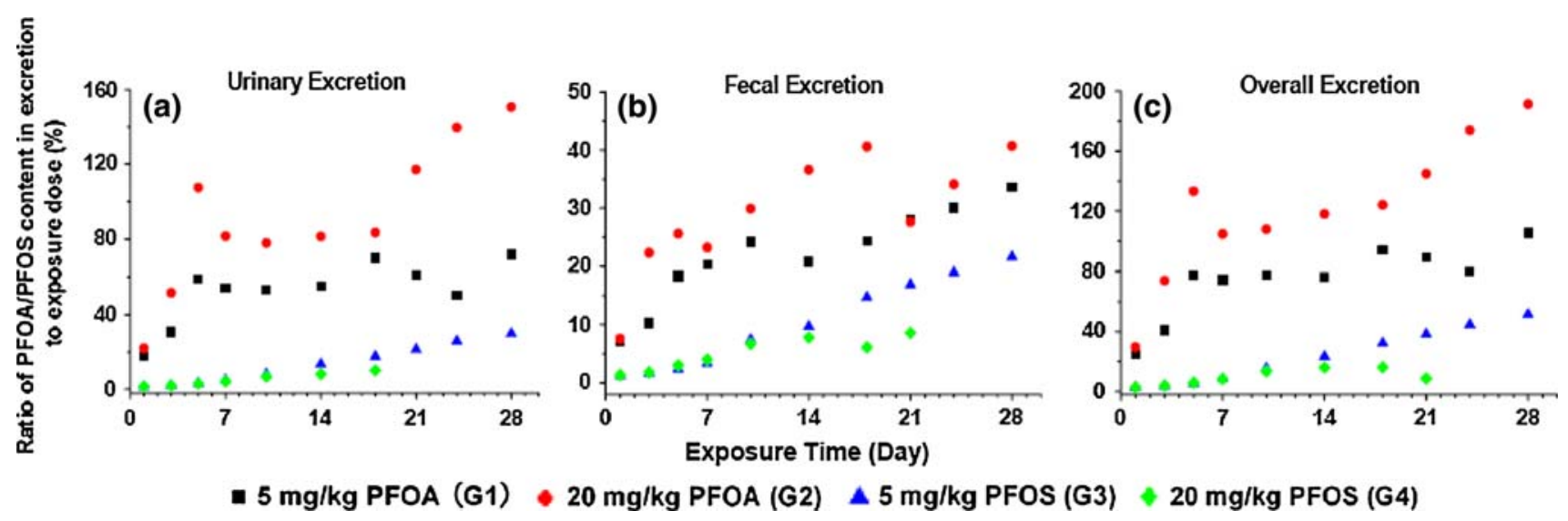

Fig. 2 Comparison of the ratios (\% per day) of the mean amounts of the target chemicals (PFOA and PFOS) in urinary (a), fecal (b), and overall (c) excretion of rats in the different treatment groups to the daily exposure doses at the appointed times (Days 1, 3, 5, 7, 10, 14, $18,21,24$, and 28) throughout the exposure period. Data are expressed as mean $\pm \operatorname{SE}(n \geq 5)$ the amounts of absorbed toxicants can be indirectly deduced from the fecal excretion. As indicated in Fig. 2b, the mean daily fecal excretion rates of PFOA in G1 and G2 were $7.2 \%$ and $7.7 \%$, respectively, in the first 24-h dosing, indicating that at least $92.8 \%$ and $92.3 \%$ of the dosed PFOA amounts were absorbed by the male rats. Apparently there was no significant difference between the high- and the low-dose groups in the first 24-h fecal elimination. Similarly, rapid absorption of PFOA has been reported by Gibson and Johnson (1979), who found that male rats absorbed $93 \%$ of an oral dose within $24 \mathrm{~h}$. Additionally, the mean fecal elimination rates of PFOA in G1 were mostly comparable to those in G2. It was thus concluded that PFOA was eliminated through feces in a dose-related manner during the subchronic exposure.

\section{Total Excretion}

On the basis of the results for urinary and fecal excretion, the daily total amounts of PFOA excreted in all groups were summarized in Fig. 1a, b by calculating the sums of the values for urine and feces, and an integrative condition of excretion is reflected. For both $\mathrm{G} 1$ and G2, the change in total excretion of PFOA was closer to that in urinary excretion rather than fecal excretion, because amounts of PFOA were much greater in urine than in feces. Therefore, urinary excretion was considered to be the principal excretion route, with respect to the higher amounts of PFOA excreted through the urine than through the feces. For the average overall excretion rates (Fig. 2c), except for the values on Day 1 in G1 and G2 (24.7\% vs. $29.6 \%$ per day), which were comparable, the values in G2 were universally higher than those in G1 on the following days, indicating a faster rate of PFOA excretion at a relatively high dose $(20 \mathrm{mg} / \mathrm{kg}$ body weight $[\mathrm{bw}] /$ day $)$.

Elimination of PFOS in Rats

\section{Urine}

The changes in the average PFOS contents in urinary excretion measured in the male rats in two PFOS dose groups (G3 and G4) are represented in Fig. 1c, d. A continuous ascent during the 28-day dosing period was observed in urinary excretion in G3, and PFOS contents varied from the minimum of $0.018 \mathrm{mg}$ on Day 1 to the maximum of $0.505 \mathrm{mg}$ on Day 28. A similar upward trend was found in G4 at the early stage of exposure, followed by an abrupt decline from the third week, when death occurred in the rats in this group (from Day 11). The values for urinary excretion in G4 were in the range of $0.080 \mathrm{mg}$ on Day 1 (minimum) to $0.673 \mathrm{mg}$ on Day 14 (maximum). In fact, urine samples were hardly collected from Day 18, owing to the minimal drinking and urination by the rats. Thus, the mean PFOS contents in urinary excretion in G4 were evaluated with regard to urine samples collected from the surviving rats at the selected timepoints. According to our previous study (Cui et al. 2009), besides the significant ethological abnormality, the remarkably decreasing food uptake induced both a decrease in body weight of rats in G4 and a reduction in amounts of PFOS excreted in the later stages of the test, which was responsible for the high accumulation of PFOS in the rats. There was no doserelated urinary elimination of PFOS during the first 2 weeks, and no apparent difference in urinary excretion rates of PFOS between the low- and the high-dose groups (Fig. 2a). However, only about $1.4 \%$ and $1.5 \%$ of the initial 
doses of PFOS were excreted through the urine in G3 and G4 on the first day, respectively, and both groups of values increased linearly and steadily with exposure time at the relatively low level.

\section{Feces}

Figure 1c, d reflects the average fecal excretion amounts of PFOS in the two PFOS exposure groups (G3 and G4). Like urinary excretion, an upward trend existed in fecal excretion in G3, with the lowest mean amount of PFOS excreted in feces on the first day (Day 1, $0.015 \mathrm{mg}$ ) and the highest on the last day of exposure (Day 28, $0.355 \mathrm{mg}$ ). A similar increase in PFOS contents excreted in feces was observed in G4 before the death of rats occurred on Day 11, whereas, different from urinary excretion, fecal excretion of PFOS in G4 obtained a maximum (0.519 $\mathrm{mg})$ on Day 18 , reaching a relatively steady state. As a matter of fact, despite the mean PFOS amounts increasing slowly, the average concentrations of PFOS in feces were rather high in the later stages of exposure: $76.6 \pm 27.8,79.9 \pm 18.5$, and $141.6 \pm$ $7.3 \mathrm{mg} / \mathrm{kg}$ on Day 18, Day 21, and Day 24, respectively. As mentioned above, a potential reason was that the gross weights of feces collected daily decreased remarkably due to the decreasing food uptake. Therefore, for fecal excretion in G4, only the values in the first 2 weeks were available. Additionally, there was a dose-related fecal elimination of PFOS during this period, and the mean fecal excretion rates of PFOS in the two dose groups were comparable (Fig. 2b). Approximately $1.2 \%$ and $1.3 \%$ of the oral doses were eliminated by fecal excretion in G3 and G4 on Day 1, indicating that the absorption of PFOS in male rats was more than $98.8 \%$ and $98.7 \%$, respectively. Similarity of fecal excretion between the two PFOS dose groups was thereby revealed.

\section{Total Excretion}

For overall mean amounts of PFOS excreted (Fig. 1c, d), a steady upward trend was found in G3 and G4 (only in the first 2 weeks), as a result of well-regulated urinary and fecal elimination. It is noteworthy that during the first postdose day (Day 1), the low overall amounts of PFOS excreted, $0.033 \mathrm{mg}$ in G3 and $0.153 \mathrm{mg}$ in G4 (Fig. 1c, d), correspondingly resulted in lower elimination values, $2.6 \%$ and $2.7 \%$, after treatment for $24 \mathrm{~h}$ (Fig. 2c). However, urinary excretion was also considered to be the principal excretion route, as more PFOS was excreted through the urine than the feces. Like the upward trend for urine and feces, the overall excretion rates of PFOS increased in G3 (Fig. 2c), ranging from $2.6 \%$ to $23.2 \%$ per day prior to Day 14 , as did those in $\mathrm{G} 4$, ranging from $2.7 \%$ to $16.2 \%$ per day. Consequently, there was no notable difference in total excretion between the two PFOS dose groups in the first 2 weeks.

\section{Comparison of Elimination of PFOA Versus PFOS in Rats}

The statistical results for the contents of the two PFCs excreted through the urine and feces in the different exposure groups (Fig. 1) showed that the values for urinary excretion were more or less greater than those for fecal excretion throughout the test period, particularly for PFOA. One possible reason is that the organic anion transporters (OATs) rOAT2 and rOAT3 are responsible for the renal excretion of these chemicals in rats (Kudo et al. 2002). This was also in agreement with the results of other research groups, which showed that excretion of PFCs occurred through urine and feces in animals, wherein urinary excretion was the primary elimination route for both PFOA and PFOS (Butenhoff et al. 2004; Kudo and Kawashima 2003). By this token, renal elimination is critical for detoxification of PFOA and PFOS.

Previous studies have documented that both PFOA and PFOS are easily and rapidly absorbed in the gastrointestinal tract. In general, the intestine is the first barrier encountered by exogenous chemicals before eventually entering the circulatory system and being distributed through the body toward target organs, where they can exert their toxicity. Therefore, the terminal concentrations in whole blood of the treated groups on Day 28 [data published in our previous study, Cui et al. 2009) are very important for comparison of the results and, accordingly, are reported here (Fig. 3), together with the concentrations

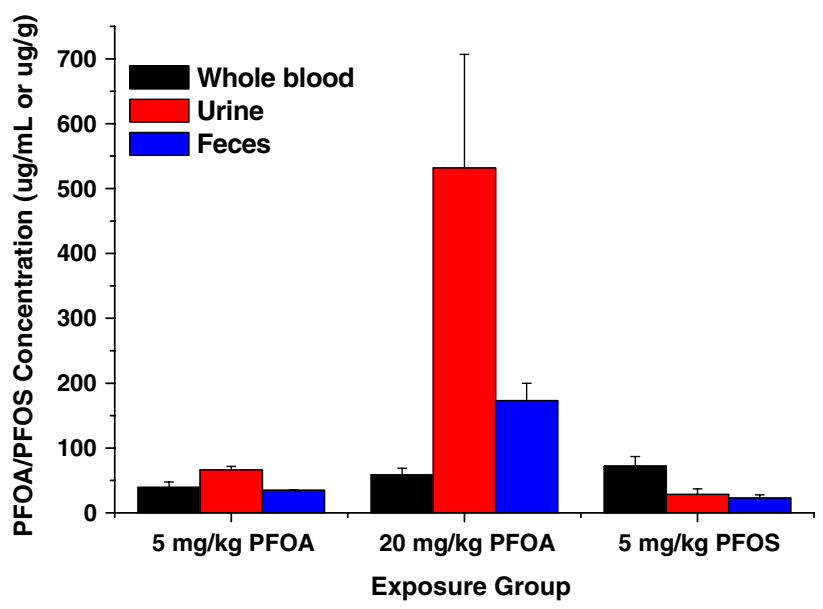

Fig. 3 Comparison of the average concentrations in whole blood (data cited from our previous paper, Cui et al. 2009), urine, and feces of rats in different treatment groups on the last day of administration (Day 28). Data are expressed as mean $\pm \mathrm{SE}(n \geq 5)$. No blood, urine, or fecal samples were obtained for G4 because all 10 rats in this dose group died successively within 24 days of exposure 
of PFCs in the urine and fecal samples on the last exposure day. Figure 3 shows that the average concentrations of PFOA in the whole blood were lower than those in the urine of rats exposed to a low or high dose of PFOA on Day 28, while the situation in the low-dose PFOS group was the opposite. Additionally, the average concentrations of PFOA in blood in different dose groups (G1 and G2) were comparable, and no dose-related accumulation of PFOA was found in our previous research (Cui et al. 2009); nevertheless, PFOA levels in both urinary and fecal excretion were clearly different between G1 and G2. At the same time, the mean concentration of the target chemical in the whole blood in G3 was the highest among the three groups, whereas that in urine or feces in this group was the lowest.

Comparison of the average (urinary, fecal, and overall) elimination rates of PFOA versus PFOS (Fig 2) showed that much more PFOA was excreted by rats exposed to the same doses, especially at a relatively high dose. In detail, for both the low and the high dose, the excretion rates of PFOA were generally higher than those of PFOS, especially on the first day. The elimination rates on the first exposure day were $24.7 \%, 29.6 \%, 2.6 \%$, and $2.8 \%$ in G1, G2, G3, and G4, respectively; thus, the fastest elimination was found in G2 (20 mg PFOA/kg bw/day) and the slowest was in G3 (5 mg PFOS/kg bw/day). On the whole, compared with PFOS, PFOA shows a faster elimination rate and lower bioaccumulation, thereby leading to lower toxicities than PFOS as observed previously (Cui et al. 2009). According to current research (Kudo et al. 2002), the serum elimination half-life of PFOA is approximately 5.68 days in male rats. In contrast, an average serum elimination half-life of 7.5 days in male rats was reported after administration of PFOS, and the urinary and fecal excretion half-life was estimated to be longer than 90 days (Johnson et al. 1979). Comparatively, PFOA has a faster rate of elimination than PFOS in rats, as well as other animals and even humans. For both genders of cynomolgus monkeys, an elimination half-life of about 20-30 days was found following dosing with PFOA (Butenhoff et al. 2004), while a half-life of approximately 100-200 days was determined after exposure to PFOS (Seacat et al. 2002). Based on recent monitoring of retired production workers, it has been demonstrated that both these perfluorochemicals have a relatively longer serum elimination half-life in humans ( $4.4 \pm 3.6$ years for PFOA vs. $8-9$ years for PFOS) than in most mammals (Burris et al. 2002; Olsen et al. 2007).

In conclusion, the combined properties of rapid absorption and poor elimination contribute to the high bioaccumulation of PFOS and PFOA during repeated exposure, thus leading to observable toxicities.
Acknowledgments This work was jointly supported by the Chinese Academy of Sciences (KZCX2-YW-420-21), the National Natural Science Foundation of China $(20737003,20677068)$ and the National Science and Technology Ministry of China (2006BAK02A06-2).

\section{References}

Austin ME, Kasturi BS, Barber M, Kannan K, MohanKumar PS, MohanKumar SMJ (2003) Neuroendocrine effects of perfluorooctane sulfonate in rats. Environ Health Perspect 111:1485-1489

Biegel LB, Hurtt ME, Frame SR, O'Connor JC, Cook JC (2001) Mechanisms of extrahepatic tumor induction by peroxisome proliferators in male CD rats. Toxicol Sci 60:44-55. doi:10.1093/ toxsci/60.1.44

Burris JM, Lundberg JK, Olsen G, Simpson C, Mandel J (2002) Interim report No. 2. Determination of serum half-lives of several fluorochemicals. 3M Company, St. Paul, MN. USEPA Public Docket AR-226-1086

Butenhoff J, Costa G, Elcombe C, Farrar D, Hansen K, Iwai H, Jung R, Kenney G, Lieder P, Olsen G, Thomford P (2002) Toxicity of ammonium perfluorooctanoate in male cynomolgus monkeys after oral dosing for 6 months. Toxicol Sci 69:244-257. doi:10.1093/ toxsci/69.1.244

Butenhoff JL, Kennedy GL, Hinderliter PM, Lieder PH, Jung R, Hansen KJ, Gorman GS, Noker PE, Thomford PJ (2004) Pharmacokinetics of perfluorooctanoate in cynomolgus monkeys. Toxicol Sci 82:394-406. doi:10.1093/toxsci/kfh302

Cui L, Zhou Q, Liao C, Fu J, Jiang G (2009) Studies on the toxicological effects of PFOA and PFOS on rats using histological observation and chemical analysis. Arch Environ Contam Toxicol 56:338-349. doi:10.1007/s00244-008-9194-6

Gibson SJ, Johnson JD (1979). Absorption of FC-143-14C in rats after a single oral dose. Riker Laboratories, Inc., subsidiary of 3M Company, St. Paul, MN. USEPA Public Docket AR-2260455. USEPA, Washington, DC

Giesy JP, Kannan K (2001) Global distribution of perfluorooctane sulfonate in wildlife. Environ Sci Technol 35:1339-1342. doi: $10.1021 / \mathrm{es} 001834 \mathrm{k}$

Giesy JP, Kannan K (2002) Perfluorochemical surfactants in the environment. Environ Sci Technol 36:147A-152A. doi:10.1021/ es022253t

Guruge KS, Yeung LWY, Yamanaka N, Miyazaki S, Lam PKS, Giesy JP, Jones PD, Yamashita N (2006) Gene expression profiles in rat liver treated with perfluorooctanoic acid (PFOA). Toxicol Sci 89:93-107. doi:10.1093/toxsci/kfj011

Hanhijärvi H (1988) A proposed species difference in the renal excretion of perfluorooctanoic acid in the beagle dog and rat. In: Beynen AC, Solleveld HA (eds) New developments in biosciences: their implications for laboratory animal science. Martinus Nijhoff, Dordrecht, pp 409-412

Hansen KJ, Clemen LA, Ellefson ME, Johnson HO (2001) Compound-specific, quantitative characterization of organic fluorochemicals in biological matrices. Environ Sci Technol 35:766770. doi:10.1021/es001489z

Johnson JD, Ober RE (1980) Extent and route of excretion and tissue distribution of total carbon-14 in male and female rats after a single IV dose of FC-143-14C. Riker Laboratories, Inc., St. Paul, MN. USEPA Public Docket AR-226-0457. USEPA, Washington, DC

Johnson JD, Gibson SJ, Ober RE (1979) Extent and route of excretion and tissue distribution of total carbon-14 in rats after a single intravenous dose of FC-95-14C. Project No. 8900310200. Riker Laboratories, Inc., St. Paul, MN. USEPA Docket No. 8(e) HQ$1180-00374$ 
Kannan K, Corsolini S, Falandysz J, Fillmann G, Kumar KS, Lganathan BG, Mohd MA, Olivero J, Van Wouwe N, Yang JH, Aldous KM (2004) Perfluorooctanesulfonate and related fluorochemicals in human blood from several countries. Environ Sci Technol 38:4489-4495. doi:10.1021/es0493446

Kemper RA (2003) Perfluorooctanoic acid: toxicokinetics in the rat. DuPont Haskell Laboratories. Laboratory Project ID: DuPont7473. USEPA Administrative Record AR-226.1499

Kennedy GL, Butenhoff JL, Olsen GW, O'Connor JC, Seacat AM, Perkins RG, Biegel L, Murphy SR, Farrar D (2004) The toxicology of perfluorooctanoate. Crit Rev Toxicol 34:351-384. doi:10.1080/10408440490464705

Kudo N, Kawashima Y (2003) The toxicity and toxicokinetics of perfluorooctanoic acid in humans and animals. J Toxicol Sci 28:49-57. doi:10.2131/jts.28.49

Kudo N, Suzuki E, Katakura M, Ohmori K, Noshiro R, Kawashima Y (2001) Comparison of the elimination between perfluorinated fatty acids with different carbon chain length in rats. Chem Biol Interact 134:203-216. doi:10.1016/S0009-2797(01)00155-7

Kudo N, Katakura M, Sato Y, Kawashima Y (2002) Sex hormoneregulated renal transport of perfluorooctanoic acid. Chem Biol Interact 139:301-316. doi:10.1016/S0009-2797(02)00006-6

Lau C, Butenhoff JL, Rogers JM (2004) The developmental toxicity of perfluoroalkyl acids and their derivatives. Toxicol Appl Pharmacol 198:231-241. doi:10.1016/j.taap.2003.11.031

Lau C, Anitole K, Hodes C, Lai D, Pfahles-Hutchens A, Seed J (2007) Perfluoroalkyl acids: a review of monitoring and toxicological findings. Toxicol Sci 99:366-394. doi:10.1093/toxsci/kfm128

Luebker DJ, Hansen KJ, Bass NM, Butenhoff JL (2002) Interactions of fluorochemicals with rat liver fatty acid-binding protein. Toxicology 176:175-185. doi:10.1016/S0300-483X(02)00081-1

Newsted JL, Beach JL, Gallagher S, Giesy JP (2006) Pharmacokinetics and acute lethality of perfluorooctanesulfonate (PFOS) to juvenile mallard and northern bobwhite. Arch Environ Contam Toxicol 50:411-420. doi:10.1007/s00244-005-1137-x

Olsen GW, Burris JM, Ehresman DJ, Froehlich JW, Seacat AM, Butenhoff JL, Zobel LR (2007) Half-life of serum elimination of perfluorooctanesulfonate, perfluorohexanesulfonate, and perfluorooctanoate in retired fluorochemical production workers. Environ Health Perspect 115:1298-1305

Ren H, Vallanat B, Nelson DM, Yeung LW, Guruge KS, Lam PK, Lehman-McKeeman LD, Corton JC (2008) Evidence for the involvement of xenobiotic-responsive nuclear receptors in transcriptional effects upon perfluoroalkyl acid exposure in diverse species. Reprod Toxicol. doi: 10.1016/j.reprotox.2008. 12.011

Seacat AM, Thomford PJ, Hansen KJ, Olsen GW, Case MT, Butenhoff JL (2002) Subchronic toxicity studies on perfluoroctanesulfonate potassium salt in cynomolgus monkeys. Toxicol Sci 68:249-264. doi:10.1093/toxsci/68.1.249

Seacat AM, Thomford PJ, Hansen KJ, Clemen LA, Eldridge SR, Elcombe CR, Butenhoff JL (2003) Sub-chronic dietary toxicity of potassium perfluorooctanesulfonate in rats. Toxicology 83:117-131. doi:10.1016/S0300-483X(02)00511-5

Tan Y, Clewell HJ, Andersen ME (2008) Time dependencies in perfluorooctylacids disposition in rat and monkeys: a kinetic analysis. Toxicol Lett 177:38-47. doi:10.1016/j.toxlet.2007. 12.007

Vanden HJ, Davis J, Sommers R, Peterson R (1992) Renal excretion of perfluorooctanoic acid in male rats: inhibitory effect of testosterone. Biochem Toxicol 7:31-36. doi:10.1002/jbt.25700 70107

Ylinen M, Kojo A, Hanhijärvi H, Jaakonaho J, Peura P (1989) Stimulation by oestradiol of the urinary excretion of perfluorooctanoic acid in the male rat. Pharmacol Toxicol 65:274-277. doi:10.1111/j.1600-0773.1989.tb01172.x

Ylinen M, Kojo A, Hanhijärvi H, Peura P (1990) Disposition of perfluooctanoic acid in the rat after single and subchronic administration. B Environ Contam Toxicol 44:46-53. doi: 10.1007/BF01702360

Yoo H, Guruge KS, Yamanaka N, Sato C, Mikami O, Miyazaki S, Yamashita N, Giesy JP (2009) Depuration kinetics and tissue disposition of PFOA and PFOS in white leghorn chickens (Gallus gallus) administered by subcutaneous implantation. Ecotoxicol Environ Saf 72:26-36. doi:10.1016/j.ecoenv.2007. 09.007 